Check for updates

Cite this: Chem. Commun., 2020, 56, 5135

Received 3rd February 2020,

Accepted 28th March 2020

DOI: $10.1039 / \mathrm{d} 0 \mathrm{cc} 00882 \mathrm{f}$

rsc.li/chemcomm

\section{Conformational enhancement of fidelity in toehold-sequestered DNA nanodevices $\uparrow$}

\author{
Antoine Bader and Scott L. Cockroft (D) *
}

The operation of DNA nanodevices is often limited by erroneous strand displacement. Here we demonstrate simple design principles that reduce such leakage by up to two orders of magnitude. Enhanced operational robustness against multiple spurious inputs was obtained by simply relocating toehold overhangs to external locations and strengthening adjacent G-C clamping.

The unique properties of DNA molecules allow the engineering of complex information-processing networks. Toehold-mediated strand-displacement reactions often constitute the mechanism of choice for the operation of DNA nanodevices. ${ }^{1}$ Strand displacement involves two fully or partially complementary strands hybridising together, leading to the subsequent dehybridisation of one or more auxiliary strands. ${ }^{2}$ It is facilitated by complementary toehold regions that act as a recognition site for the binding of a strand that initiates a subsequent strand-displacement process. Strand-displacement reactions have been exploited in DNA logic, ${ }^{3}$ DNA-based chemical reaction networks ${ }^{4}$ and neural networks, ${ }^{5}$ as well as molecular switches and machines. ${ }^{2 a, 6}$ A major issue often encountered in strand-displacement-based systems is leakage, which occurs when a response is produced in the absence of the appropriate input. To tackle this problem, duplex stability can be increased by using G-C clamps at the duplex termini and the addition of magnesium ions to the media. Furthermore, gel electrophoresis allows the separation of a DNA complex of interest from a pool of structurally defective assemblies that could be prone to leak. ${ }^{3 b}$ An alternative strategy that may be employed is the use of a downstream threshold gate to consume the leak. ${ }^{3 g, 7}$ In such an approach, the threshold gate provides a thermodynamic trap that rapidly binds the leaked signal strands, meaning that a downstream gate may only be activated once a pre-determined signal threshold concentration is exceeded. Other strategies include the use of mismatches, ${ }^{8}$ remote toeholds, ${ }^{9}$

EaStCHEM School of Chemistry, University of Edinburgh, Joseph Black Building, David Brewster Road, Edinburgh EH9 3FJ, UK. E-mail: scott.cockroft@ed.ac.uk $\dagger$ Electronic supplementary information (ESI) available: Details of simulations, materials, methods and DNA sequences. See DOI: 10.1039/d0cc00882f entropic effects, ${ }^{10}$ and even unnatural modifications ${ }^{11}$ to control hybridisation kinetics, specificity, or the spatial segregation of DNA components within a circuit. ${ }^{12}$

Mechanical deactivation of toeholds constitutes another method for regulating strand-displacement reactions. In this case, the toehold required for the strand-displacement reaction is mechanically sequestered in a duplex and is only revealed after the introduction of a specific stimulus. For example, controllable activation and regulation of toeholds has been achieved by simple strand-displacement reactions, ${ }^{3 b, 13}$ allosteric reconfiguration, ${ }^{14}$ and photochemical regulation. ${ }^{15}$

Here we present a systematic approach for reducing the leakage of toehold-sequestered DNA devices. We hypothesized that secondary structures and duplex-breathing dynamics were

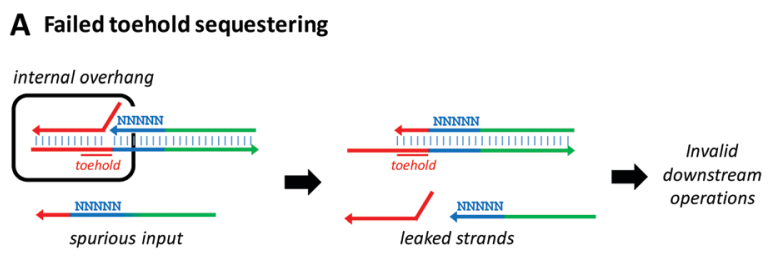

B Robust toehold sequestering

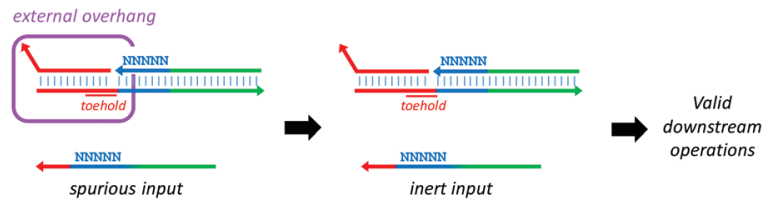

Fig. 1 Operational fidelity of a toehold-sequestered device. (A) Leakage may occur when a weakly bound toehold-sequestering strand (red) fails to prevent a spurious input strand from invading the duplex via toehold recognition, resulting in undesired release the output (leakage). (B) Robust sequestering of the toehold prevents the spurious input from triggering the device and releasing the output strand. In the present work, we examine whether the operational fidelity of such devices can be enhanced by relocating the overhang of a toehold-sequestering strand from an internal to an external position. 
A

Internal overhang

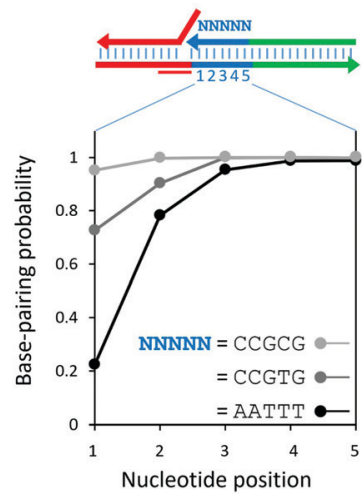

B External overhang

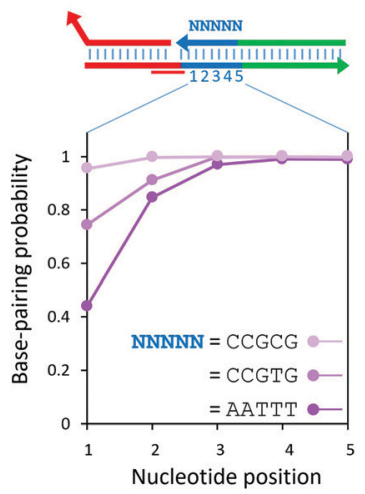

Fig. 2 Probability of base-pairing between two complementary bases predicted by NUPACK.

the main determinants of leakage in such devices. In particular, that leakage arises from failure of the toehold-sequestering strand to form a stable duplex within the "protected" toehold region, leading to unwanted release of the output (Fig. 1). We assembled devices in which the overhang on the toeholdsequestering strand was either located at the nick site (internal overhang, Fig. 1A) or at the strand terminus (external overhang, Fig. 1B). We tested the hypothesis that the location of the toehold overhang was critical to the fidelity of the stranddisplacement process by examining the conformational properties of DNA assemblies containing internal $v s$. external overhangs using the NUPACK software package (Fig. 2 ), ${ }^{16}$ OxDNA molecular dynamics simulations (Fig. 3), ${ }^{17}$ and an electrophoretic gel mobility assay (Fig. 4). A general improvement in the fidelity of toehold sequestered devices by repositioning the toehold overhang was demonstrated by testing the assemblies against a challenging series of fully complementary spurious inputs with different toehold binding energies (Table S1, ESI $\dagger$ ) and by varying the sequence flanking the nick site (Fig. 5).

DNA molecules can undergo spontaneous local reconfiguration at temperatures below the DNA melting temperature. We hypothesised that this process, defined as DNA breathing, ${ }^{18}$ may transiently reveal a few bases of a toehold-binding domain, leading to unwanted strand invasion and premature strand-displacement of an output (leakage). We further speculated that the presence of an internal overhang (Fig. 1A) could be detrimental to the overall stability of the toehold-sequestered duplex region, and thus induce leakage. Such an influence could be manifested through steric or electrostatic repulsion, or a combination of both. The online nucleic acid design tool, NUPACK was used to evaluate the probability of fraying at the NNNNN region adjacent to the nick site (Fig. 1 and 2). ${ }^{19}$ NUPACK implements an empirical model that describes nucleic acid secondary structures. It relies on DNA nearest-neighbour parameters derived from numerous UV melting experiments to describe DNA polymers and oligomers. ${ }^{20}$

NUPACK indicated that the probability of base pairing in the NNNNN region increased when the overhang was relocated

from an internal position to an external position, even when the duplex-forming sequences were otherwise identical (Fig. 2A vs. 2B). This effect is particularly striking in the absence of G-C clamps $($ NNNNN $=$ AATTT $)$, since any additional base-pair stabilisation appeared unable to balance the destabilisation induced by the presence of the overhang. Base-pairing probability was predicted to increase substantially the further the base-pair was positioned away from the nick site. Adding G-C clamps adjacent to the nick site (NNNNN = CCGTG; CCGCG) increased base-pairing probability for both internal and external overhang structures. These calculations suggest that strong G-C clamping could mitigate the destabilising effect of an internal overhang, and thus reduce leakage. Additionally, these results suggest that relocating the overhang from an internal to an external position could also help to tackle leakage.

To further investigate and compare the stability of the assemblies presenting an internal or external overhang, coarse-grained molecular dynamics (MD) simulations were performed using oxDNA. ${ }^{17 b, 21}$ The simulations found that the external-overhang assemblies adopted a linear, fully stacked conformation across the nick site (arrows in Fig. 3B), whereas the internal-overhang assemblies were kinked at the nick site (arrows in Fig. 3A). The added flexibility in the internal-overhang devices results from destabilisation of the duplex through repulsive steric and electrostatic influences, resulting in the loss of stacking interactions. Moreover, these simulations also revealed the possibility of duplex breathing at the nick site, which would lead to transient unravelling of nucleotides that might act as a site for the undesired initiation of strand displacement.

Further evidence for the added flexibility of internal-overhang devices compared to external-overhang devices was provided by polyacrylamide gel electrophoretic mobility shift assays performed under both native and denaturing conditions (Fig. 4A and B, respectively). ${ }^{22}$ Under native conditions, internal- and externaloverhang assemblies presented similar mobilities, owing to the stabilising effect of magnesium ions on the stacked conformation. The slight retardation of internal-overhang structures can be rationalised as arising from a kinked conformation.
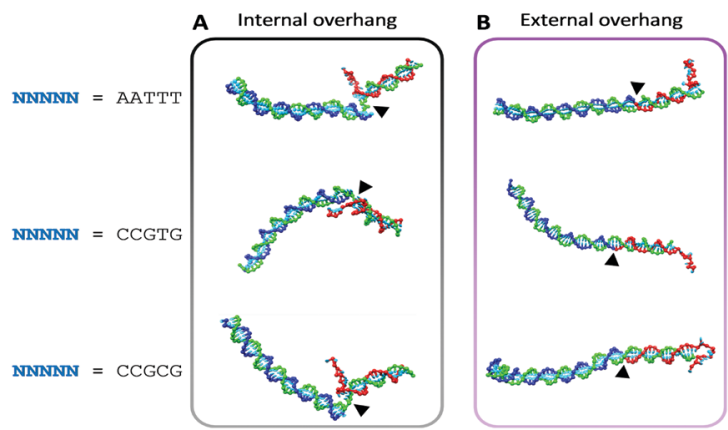

Fig. 3 Minimised structures of the toehold-sequestered devices determined using oxDNA molecular dynamics simulations. Base stacking at the nick location (black arrows) is disrupted by an internally overhanging sequestering strand (red), which perturbs the conformation of the DNA structures compared to those with an external overhang. Sequences are provided in the ESI. $\dagger$ 
This retardation is increased in the presence of urea, which is known to increase the bending of a dsDNA fragment at the nick site. $^{22}$ This causes a decrease in the mobility of the internaloverhang species due to reduced stabilisation from coaxial stacking. In contrast, the mobility of each of the externaloverhang assemblies was identical, consistent with the stacked conformation of these structures being conserved, irrespective of the presence of urea.

The robustness of all toehold-sequestered devices was then assessed experimentally by challenging them with a series of fully sequence-matched spurious inputs of different lengths (Fig. 5). Gates were first annealed $\left(90{ }^{\circ} \mathrm{C}\right.$ for $5 \mathrm{~min}$ followed by slow cooling to $5{ }^{\circ} \mathrm{C}$ over $2 \mathrm{~h}$ ), and subsequently incubated (room temperature for $18 \mathrm{~h}$ ) with deactivated inputs lacking the toeholds required for strand displacement. These steps eliminated the formation of incorrectly assembled gates. ${ }^{3 b}$ The correctly assembled gates were then purified by gel electrophoresis before being challenged by the addition of spurious input sequences. Native polyacrylamide gel electrophoresis and staining was used to quantify any resulting leakage (Fig. S2 and Tables S2, S3, ESI $\dagger$ ). Interrogation of a gate by a given spurious input was repeated four times on four different gels to evaluate the standard deviation for each dataset. Analysis of gel band intensities was permitted by the similarity of the lengths of the different strands. ${ }^{8 b}$ This allowed the observed leakage yield arising from unwanted displacement of the output by the spurious input to be determined. The leakage yield was calculated as $\chi_{n}=\left[X_{n}\right] /\left(\left[X_{n}\right]+\left[D_{n}\right]\right)$, where $\left[X_{n}\right]$ and $\left[D_{n}\right]$ are the band intensities of the leak-induced duplex product $X_{n}$ and the intact toehold-sequestered device $D_{n}$, respectively. The data presented in Fig. 5 shows that the external-overhang structures were always more resistant to spurious input invasion compared to internal-overhang structures (black/grey vs. purple). This demonstrated the decisive importance of the positioning of the toehold-sequestering strand overhang.

Indeed, the presence of the overhang at the nick site is detrimental to the stability of the assembly and reduces the stacking interactions between the adjacent strands due to the sterically induced kinked conformation. Such instability reveals an undesired, transient toehold at the nick position. Overall,
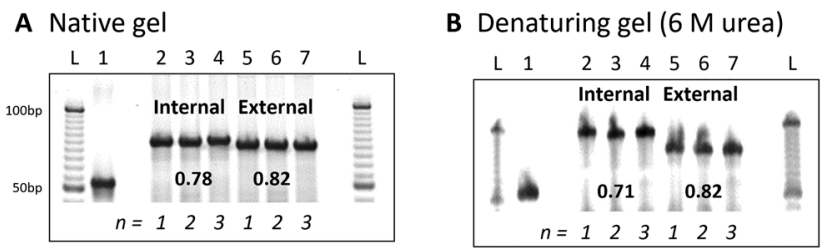

Fig. 4 Electrophoretic mobility shift assay under (A) native and (B) $6 \mathrm{M}$ urea-denaturing conditions showing increased mobility of three different external-overhang assemblies ( $L 5$ to L7) relative to three different assemblies with internal overhangs ( $L 2$ to L4). Relative mobility ratios (0.71-0.82) indicated below the bands were calculated by dividing the migration distance of the band relative to a 55bp duplex internal standard. The variation of mobility in internal-overhang species results from increased destabilisation at the nick site in the presence of urea. Such an effect is not observed for external-overhang species, indicative of a fully stacked, and therefore less flexible conformation. Sequences are provided in the ESI. $\dagger$ shifting the overhang from the internal nick site to the duplex terminus decreased the leakage by a factor of up to 26. Moreover, the amount of leak generally decreased as the length and therefore specificity of the spurious input decreased (series of Input $\mathrm{a}_{\mathrm{a}}$ to Input $_{\mathrm{d}}$ ). Deletion of five bases at the 3 '-end of the input strand (Inputs $_{\mathrm{b}-\mathrm{d}} v s$. Input $\mathrm{a}_{\mathrm{a}}$ ) converts the displacement mechanism from a full strand displacement to a toehold-exchange process, ${ }^{23}$ which further reduces the thermodynamic favourability of the hybridisation between spurious input and target. Finally, increasing the stability of the NNNNN-containing duplex adjacent to the nick site also reduced leakage by maintaining duplex integrity though a combination of stabilising H-bonding and stacking interactions. The combination of relocating the overhang to an external site, G-C clamping adjacent to the nick site, and reducing the length of the spurious inputs reduced leakage from $28 \%$ for the leakiest device to $0.3 \%$ for the most robust one, with the secondary structure appearing to be a key factor in enhancing the fidelity of such devices. One limitation of the data presented in Fig. 5 is that the method employed only provides leakage yields at the end of a set time-course; real-time monitoring of displacement kinetics provides the most thorough means of quantifying strand displacement. $^{23}$

In conclusion, we have used computational modelling and experimental gel-shift assays to examine conformational influences on the fidelity of toehold-sequestered DNA nanodevices that operate via strand-displacement mechanisms. The conformational stability of DNA assemblies was demonstrated to be critical to the extent of unwanted strand leakage. The presence of an overhang at the nick position induces a conformational change that disrupts stacking interactions and destabilises the assembly, making it less robust to unwanted strand invasion. Thus, relocating the toehold overhang from an internal to an external location decreased leakage by a factor of up to 26 . Indeed, toehold relocation was found to play a dominant role in improving device fidelity compared to variation of either the spurious input or clamping sequence. Furthermore, combining

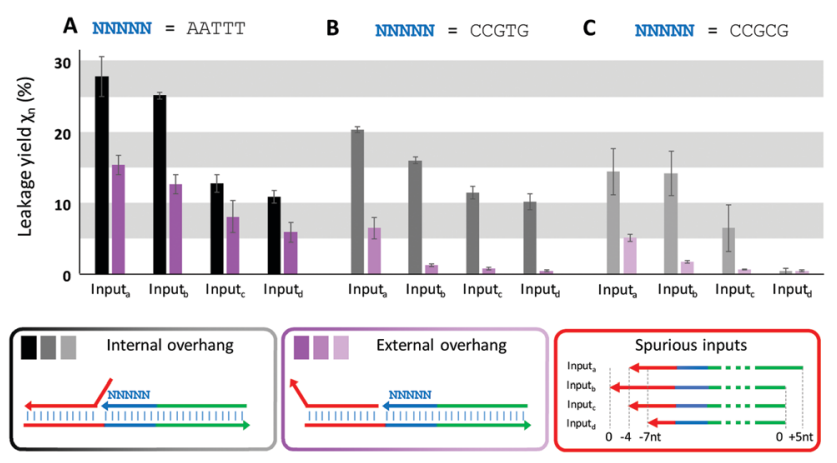

Fig. 5 Spurious input-induced leakage in internal (black/grey) and external (purple/pink) toehold-sequestered devices determined using polyacrylamide electrophoresis (Fig. S1, S2 and Tables S2, S3, ES1†). Relocating the overhang on the toehold-sequestering strand from an internal to an external position always reduces the extent of undesired leakage, irrespective of the spurious input ( $x$-axis) or NNNNN clamping sequences (A to C). In addition, toehold location plays a dominant role in improving device fidelity compared to variation of either the input or NNNNN clamping sequence. 
the external-overhang design with shortened spurious inputs and strong G-C clamping at the nick site dramatically reduced leakage from $28 \%$ to only $0.3 \%$ in otherwise sequence-matched devices. The modularity of DNA-based devices means that these simple design principles can be widely exploited to enhance the operational fidelity of any strand-displacement-based DNA device. The relocation of toehold overhangs to external positions is particularly easy to incorporate into existing strand-displacement networks, since the information-containing target sequences do not need to be changed, just rearranged. In contrast, the benefits of shortened spurious inputs and strong G-C clamping adjacent to toehold sites necessitates that these design principles are considered at an early stage of sequence design. Our findings highlight the importance of architectural considerations in the design of robust signal-responsive DNA structures.

We thank the ERC starting grant no. 336935, for financial support.

\section{Conflicts of interest}

There are no conflicts to declare.

\section{Notes and references}

1 D. Y. Zhang and G. Seelig, Nat. Chem., 2011, 3, 103-113.

2 (a) B. Yurke and A. Mills, Jr., Genet. Program. Evol. M., 2003, 4, 111-122; (b) N. Srinivas, T. E. Ouldridge, P. Sulc, J. M. Schaeffer, B. Yurke, A. A. Louis, J. P. Doye and E. Winfree, Nucleic Acids Res., 2013, 41, 10641-10658.

3 (a) N. H. Voelcker, K. M. Guckian, A. Saghatelian and M. R. Ghadiri, Small, 2008, 4, 427-431; (b) G. Seelig, D. Soloveichik, D. Y. Zhang and E. Winfree, Science, 2006, 314, 1585-1588; (c) A. Bader and S. L. Cockroft, Chem. - Eur. J., 2018, 24, 4820-4824; (d) A. J. Genot, J. Bath and A. J. Turberfield, J. Am. Chem. Soc., 2011, 133, 20080-20083; (e) M. N. Stojanović and D. Stefanović, J. Am. Chem. Soc., 2003, 125, 6673-6676; $(f)$ M. N. Stojanovic and D. Stefanovic, Nat. Biotechnol., 2003, 21, 1069-1074; $(g)$ L. Qian and E. Winfree, Science, 2011, 332, 1196-1201.

4 (a) D. Soloveichik, G. Seelig and E. Winfree, Proc. Natl. Acad. Sci. U. S. A., 2010, 107, 5393-5398; (b) Y.-J. Chen, N. Dalchau, N. Srinivas, A. Phillips, L. Cardelli, D. Soloveichik and G. Seelig, Nat. Nanotechnol., 2013, 8, 755-762; (c) N. Srinivas, J. Parkin, G. Seelig, E. Winfree and D. Soloveichik, Science, 2017, 358, eaal2052; (d) P. J. Milnes, M. L. McKee, J. Bath, L. Song, E. Stulz, A. J. Turberfield and R. K. O'Reilly, Chem. Commun., 2012, 48, 5614-5616; (e) M. L. McKee, P. J. Milnes, J. Bath, E. Stulz, A. J. Turberfield and R. K. O'Reilly, Angew. Chem., Int. Ed., 2010, 49, 7948-7951.

5 L. Qian, E. Winfree and J. Bruck, Nature, 2011, 475, 368-372.

6 (a) F. C. Simmel and W. U. Dittmer, Small, 2005, 1, 284-299; (b) J. Bath and A. J. Turberfield, Nat. Nanotechnol., 2007, 2, 275-284; (c) A. J. Turberfield, J. C. Mitchell, B. Yurke, A. P. Mills, M. I. Blakey and
F. C. Simmel, Phys. Rev. Lett., 2003, 90, 118102; (d) N. C. Seeman and H. F. Sleiman, Nat. Rev. Mater., 2017, 3, 17068.

7 L. Qian and E. Winfree, J. R. Soc., Interface, 2011, 8, 1281-1297.

8 (a) Y. S. Jiang, S. Bhadra, B. Li and A. D. Ellington, Angew. Chem., Int. Ed., 2014, 53, 1845-1848; (b) D. Y. Zhang, S. X. Chen and P. Yin, Nat. Chem., 2012, 4, 208-214; (c) R. R. F. Machinek, T. E. Ouldridge, N. E. C. Haley, J. Bath and A. J. Turberfield, Nat. Commun., 2014, 5, 5324; (d) D. W. B. Broadwater Jr and H. D. Kim, Biophys. J., 2016, 110, 1476-1484; (e) C. Li, Y. Li, Y. Chen, R. Lin, T. Li, F. Liu and N. Li, RSC Adv., 2016, 6, 74913-74916.

9 (a) A. J. Genot, D. Y. Zhang, J. Bath and A. J. Turberfield, J. Am. Chem. Soc., 2011, 133, 2177-2182; (b) B. Wang, X. Zhou, D. Yao, X. Sun, M. He, X. Wang, X. Yin and H. Liang, Chem. Commun., 2017, 53, 10950-10953.

10 B. Wang, C. Thachuk, A. D. Ellington, E. Winfree and D. Soloveichik, Proc. Natl. Acad. Sci. U. S. A., 2018, 115, E12182.

11 (a) X. Olson, S. Kotani, B. Yurke, E. Graugnard and W. L. Hughes, J. Phys. Chem. B, 2017, 121, 2594-2602; (b) W. Ding, W. Deng, H. Zhu and H. Liang, Chem. Commun., 2013, 49, 9953-9955; (c) X. Xu and X. Yang, Chem. Commun., 2014, 50, 805-807.

12 (a) M. Teichmann, E. Kopperger and F. C. Simmel, ACS Nano, 2014, 8, 8487-8496; (b) B. M. Frezza, S. L. Cockroft and M. R. Ghadiri, J. Am. Chem. Soc., 2007, 129, 14875-14879.

13 (a) S. Nakayama, L. Yan and H. O. Sintim, J. Am. Chem. Soc., 2008, 130, 12560-12561; (b) R. M. Dirks and N. A. Pierce, Proc. Natl. Acad. Sci. U. S. A., 2004, 101, 15275-15278.

14 (a) Y. Xing, Z. Yang and D. Liu, Angew. Chem., Int. Ed., 2011, 50, 11934-11936; (b) A. Amodio, B. Zhao, A. Porchetta, A. Idili, M. Castronovo, C. Fan and F. Ricci, J. Am. Chem. Soc., 2014, 136, 16469-16472.

15 (a) A. Prokup, J. Hemphill and A. Deiters, J. Am. Chem. Soc., 2012, 134, 3810-3815; (b) J. Hemphill and A. Deiters, J. Am. Chem. Soc., 2013, 135, 10512-10518; (c) F. Huang, M. You, D. Han, X. Xiong, H. Liang and W. Tan, J. Am. Chem. Soc., 2013, 135, 7967-7973.

16 J. N. Zadeh, C. D. Steenberg, J. S. Bois, B. R. Wolfe, M. B. Pierce, A. R. Khan, R. M. Dirks and N. A. Pierce, J. Comput. Chem., 2011, 32, $170-173$.

17 (a) J. P. Doye, T. E. Ouldridge, A. A. Louis, F. Romano, P. ulc, C. Matek, B. E. Snodin, L. Rovigatti, J. S. Schreck, R. M. Harrison and W. P. Smith, Phys. Chem. Chem. Phys., 2013, 15, 20395-20414; (b) P. Šlc, F. Romano, T. E. Ouldridge, L. Rovigatti, J. P. K. Doye and A. A. Louis, J. Chem. Phys., 2012, 137, 135101.

18 (a) M. Frank-Kamenetskii, Nature, 1987, 328, 17-18; (b) M. Guéron, M. Kochoyan and J.-L. Leroy, Nature, 1987, 328, 89-92.

19 (a) X. Olson, S. Kotani, J. E. Padilla, N. Hallstrom, S. Goltry, J. Lee, B. Yurke, W. L. Hughes and E. Graugnard, ACS Synth. Biol., 2016, 6, 84-93; (b) J. N. Zadeh, C. D. Steenberg, J. S. Bois, B. R. Wolfe, M. B. Pierce, A. R. Khan, R. M. Dirks and N. A. Pierce, J. Comput. Chem., 2011, 32, 170-173.

20 (a) J. SantaLucia, Proc. Natl. Acad. Sci. U. S. A., 1998, 95, 1460-1465; (b) M. Zuker, Nucleic Acids Res., 2003, 31, 3406-3415.

21 J. P. K. Doye, T. E. Ouldridge, A. A. Louis, F. Romano, P. Sulc, C. Matek, B. E. K. Snodin, L. Rovigatti, J. S. Schreck, R. M. Harrison and W. P. J. Smith, Phys. Chem. Chem. Phys., 2013, 15, 20395-20414.

22 (a) E. Protozanova, P. Yakovchuk and M. D. Frank-Kamenetskii, J. Mol. Biol., 2004, 342, 775-785; (b) H. Kuhn, E. Protozanova and V. V. Demidov, Electrophoresis, 2002, 23, 2384-2387; (c) P. Yakovchuk, E. Protozanova and M. D. Frank-Kamenetskii, Nucleic Acids Res., 2006, 34, 564-574.

23 D. Y. Zhang and E. Winfree, J. Am. Chem. Soc., 2009, 131, 17303-17314. 